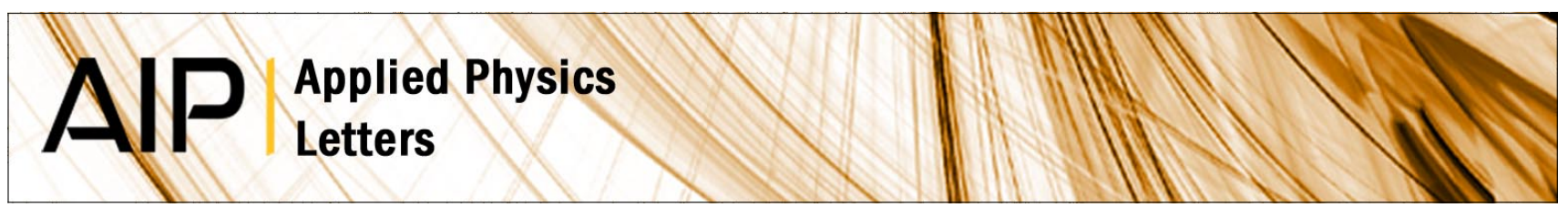

\title{
Doping of zincselenidetelluride
}

W. Faschinger, S. Ferreira, and H. Sitter

Citation: Appl. Phys. Lett. 64, 2682 (1994); doi: 10.1063/1.111490

View online: http://dx.doi.org/10.1063/1.111490

View Table of Contents: http://apl.aip.org/resource/1/APPLAB/v64/i20

Published by the American Institute of Physics.

Additional information on Appl. Phys. Lett.

Journal Homepage: http://apl.aip.org/

Journal Information: http://apl.aip.org/about/about_the_journal

Top downloads: http://apl.aip.org/features/most_downloaded

Information for Authors: http://apl.aip.org/authors

\section{ADVERTISEMENT}

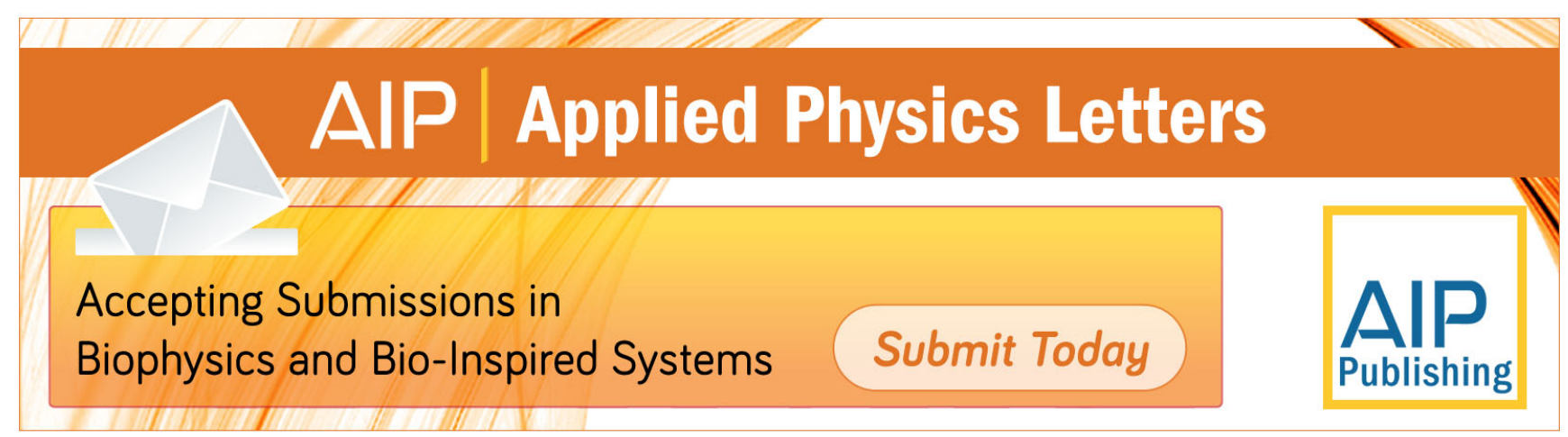




\title{
Doping of zinc-selenide-telluride
}

\author{
W. Faschinger \\ Institut für Halbleiterphysik, Universität Linz, Austria \\ S. Ferreira ${ }^{\mathrm{a})}$ and $\mathrm{H}$. Sitter \\ Institut für Experimentalphysik, Universitat Linz, Austria
}

(Received 29 November 1993; accepted for publication 28 February 1994)

\begin{abstract}
We investigate the doping behavior of $\mathrm{ZnSe} / \mathrm{ZnTe}$ short period superlattices. $p$-type doping is achieved with a dc nitrogen plasma source, $n$-type doping with chlorine from a $\mathrm{ZnCl}_{2} \mathrm{Knudsen}$ source. Even a small Te content has a strong positive effect on $p$ doping: Doping levels in the upper $10^{19} \mathrm{~cm}^{-3}$ range are achieved, and ohmic contacts can be obtained even for low carrier concentrations. The data are in excellent agreement with a theory based on the amphoteric native defect model. The opposite is valid for $n$ doping: At Te concentrations above $20 \%$ electron concentrations are below $10^{16} \mathrm{~cm}^{-3}$. As a possible way to get both good $n$ - and $p$-type doping at the same lattice constant we propose the use of the quaternary compound $\mathrm{Zn}_{(1-y)} \mathrm{Mg}_{(y)} \mathrm{Se}_{(1-x)} \mathrm{Te}_{(x)}$.
\end{abstract}

The recent breakthrough in blue semiconductor laser technology was driven by the introduction of activated nitrogen as a $p$ dopant for $\mathrm{ZnSe} .{ }^{1-4}$ However, the performance of blue laser devices suffers from the fact that the obtainable hole concentrations in $\mathrm{ZnSe}$ are still too low (in the upper $10^{7}$ range), and the contact resistance between $p$ - $\mathrm{ZnSe}$ and metals is very high. On the other hand, the hole concentrations reported for $\mathrm{ZnTe}$ doped with activated nitrogen using rf plasma are much higher (near to $10^{19}$ ), and ohmic contacts to this material can be easily obtained. ${ }^{5}$ It would be an obvious advantage if these properties could be exploited in blue laser structures. One recent attempt to do this was the use of a $\mathrm{ZnTe}$ contact layer which is connected to the $\mathrm{ZnSe}$ by a graded $\mathrm{ZnSe}_{(1-x)} \mathrm{Te}_{(x)}$ region. ${ }^{6}$ In fact this allowed the formation of ohmic contacts to $p$-ZnSe. However, the large lattice mismatch $(8 \%)$ between $\mathrm{ZnSe}$ and $\mathrm{ZnTe}$ induces the introduction of a very dense dislocation network that again severely limits device performance.

An alternative way would be the use of $\mathrm{ZnSe}_{(1-x)} \mathrm{Te}_{(x)}$ mixed crystals or $\mathrm{ZnSe} / \mathrm{ZnTe}$ short period superlattices (SLs) instead of the currently used $\mathrm{ZnS}_{(1-x)} \mathrm{Se}_{(x)}$ : Since the obtainable $p$-doping levels in $\mathrm{ZnTe}$ are much higher than in $\mathrm{ZnSe}$, one would expect that the admixture of Te has a positive effect on $p$ doping and contacting. Besides the difference in dopability, a second distinct difference between $\mathrm{ZnSe}$ and $\mathrm{ZnTe}$ is the fact that in an absolute energy scale the $\mathrm{ZnTe}$ valence band edge lies $1 \mathrm{eV}$ higher with respect to the $\mathrm{ZnSe}$ valence band edge. A similar correspondence between doping behavior and band edge positions is observed in III-V's, and the so-called amphoteric native defect model gives a quantitative correlation between these two properties. ${ }^{7}$ It has been suggested recently that this model can also be applied to wide gap II-VI materials, ${ }^{8}$ and especially the doping of $\mathrm{ZnSe} \mathrm{Te}$ and $\mathrm{ZnMgSe}$ was discussed by Chadi. ${ }^{9}$ An estimate of the effect of Te alloying can therefore be gained by determining the influence of $\mathrm{Te}$ on the position of the valence

\footnotetext{
${ }^{a}$ Permanent address: Instituto Nacional de Pesquisas Espaciais, Sao Jose dos Campos, Brazil.
}

band edge. We calculated this effect by simulating the mixed crystals with ZnSe/Zn'le short period SLs. The average Te content of the SLs was varied between $10 \%$ and $90 \%$, while the period of each SL was adapted in a way that the effectivc superlattice energy gap calculated with a Kronig-Penney type model corresponds to the known energy gap of the alloy with the same average - Te content. ${ }^{10}$ Because of this adjustment we assume that the alloy will behave accordingly. The parameters used in the calculation were effective electron and hole masses of 0.16/0.75 $m_{e}$ for $\mathrm{ZnSe}$, and 0.13/0.6 $m_{e}$ for $\mathrm{ZnTe}$. The valence band offset between $\mathrm{ZnSe}$ and $\mathrm{ZnTe}$ was assumed to be $970 \mathrm{meV} .{ }^{11}$ The advantage of this procedure is that the Kronig-Pcnney calculation gives the energy positions of the SL minibands with respect to the $\mathrm{ZnSe}$ and $\mathrm{ZnTe}$ conduction and valence bands. Figure 1 shows the resulting change of the valence and conduction band edge as a function of the Te content (symbols). The solid lines are polynomial fits through the calculated points.

It can be seen that the shift of the valence band edge is more pronounced at low Te contents. Therefore we expect an effect on $p$ doping already at moderate Te content.

In order to clarify this point experimentally, we grew a series of doped short period ZnSe/ZnTe SLs (periods between 1.5 and $2.5 \mathrm{~mm}$ ) with varying average Te content. SLs instead of mixed crystals were chosen since the molecular beam epitaxy (MBE) growth of $\mathrm{ZnSe}_{(1-x)} \mathrm{Te}_{(x)}$ mixed crystals with controlled composition is difficult because of a competition reaction between $\mathrm{Se}$ and $\mathrm{Te}$ on the surface of the growing film. ${ }^{12}$ In one sample, $15 \% \mathrm{Mg}$ were added to check the influence of $\mathrm{Mg}$ on $p$ doping. The samples were grown in a vertical $\mathrm{MBE}$ reactor equipped with $\mathrm{Cd}, \mathrm{Zn}, \mathrm{Mg}$, and $\mathrm{Te}$ effusion cells, a Se-cracker cell, a $\mathrm{ZnCl}_{2}$ source for $\mathrm{Cl}$ doping, and a dc plasma source for nitrogen doping. The dc plasma source was mounted with a sample-source distance of $5 \mathrm{~cm}$. It was operated at a power of $15 \mathrm{~W}$.

Details of the $p$ doping of $\mathrm{ZnTe}$ with this source are reported elsewhere. ${ }^{13}$ The achieved hole concentrations in $\mathrm{ZnTe}$ depend only weakly on stoichiometry, with the highest values $\left(10^{20} \mathrm{~cm}^{-3}\right)$ obtained for slightly Te stabilized conditions, as determined by the disappearance of half-order 


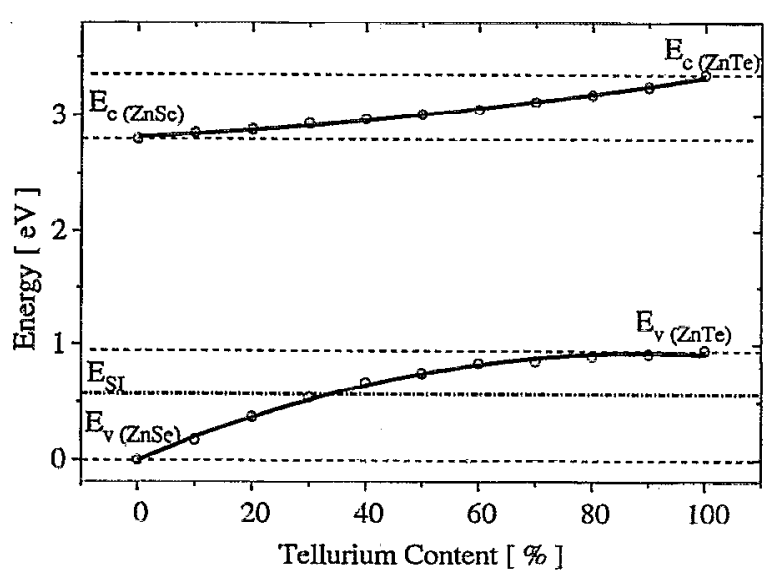

FIG. 1. Calculated position to the conduction and valence band edge of $\mathrm{ZnSe}_{(1-x)} \mathrm{Te}_{(x)}$ as a function of the tellurium content (symbols). The solid lines are polynomial fits to the calculated values. As a reference, the positions of the $\mathrm{ZnSe}$ and $\mathrm{ZnTe}$ conduction and valence bands are indicated as broken lines. The line labeled $E_{\text {SI }}$ represents a reference energy level.

RHEED (reflection high energy electron diffraction) streaks in the (001) azimuth, indicating the transition between a $c(2$ $\times 2)$ reconstruction typical for metal stabilization to a $(2 \times 1)$ reconstruction typical for an anion stabilized surface. Since such slightly anion stabilized conditions are reported to be also optimal for $p$ doping of $\mathrm{ZnSe}^{3}$ for the $\mathrm{ZnSe}$ part of the SLs the Se flux was adjusted accordingly. The substrate temperature for all samples was $280^{\circ} \mathrm{C}$.

Additionally, we investigated the doping of such SLs with $\mathrm{Cl}$. The $\mathrm{ZnCl}_{2}$ source was used under conditions optimized to obtain high doping levels in $\mathrm{ZnSe}\left(1.5 \times 10^{19}\right.$ for a source temperature of $140^{\circ} \mathrm{C}$ ). Each SL had a thickness of $0.5 \mu \mathrm{m}$ and was grown on a 0.5 -m-thick $\mathrm{ZnSe}$ buffer on (100) oriented GaAs. The average Te content of the SLs was determined by fitting the $\mathrm{x}$-ray diffraction spectrum of each SL using dynamical diffraction theory. Carrier concentrations and mobilities were measured by Hall effect in Van der Pauw geometry at room temperature.

The measured Hall mobilities for holes are rather low $\left(\approx 10 \mathrm{~cm}^{2} / \mathrm{V} \mathrm{s}\right.$ ). This value gets higher (up to $40 \mathrm{~cm}^{2} / \mathrm{N} \mathrm{s}$ ) if the doped zone is separated from the heterointerface by an undoped spacer which reduces the dislocation density. ${ }^{14}$ However, in contrast to the mobility, the measured carrier concentrations turned out to be independent of the spacer layer thickness, showing that a large dislocation density leads to low mobilities, but that dislocations are not acting as compensating centers which reduce carrier concentrations.

Figure 2 shows the achieved $p$ - and $n$-doping levels as a function of the average Te content (symbols). Although a wide stoichiometry range was investigated for pure $\mathrm{ZnSe}$ samples, the obtained hole concentrations in $\mathrm{ZnSe}$ were below the detection limit of our Hall apparatus $\left(\approx 10^{16} \mathrm{~cm}^{-3}\right)$. This could be due to two reasons: First, the plasma power possible with our dc source is an order of magnitude lower than the power used for rf plasma doping ( $15 \mathrm{~W}$ compared to $150 \mathrm{~W},{ }^{15}$ so that lower doping levels could be expected. Second, the activated nitrogen species in a dc and a if plasma need not necessarily be the same. This would also explain the fact that our ZnTe doping levcls arc an order of magnitude higher than the ones reported for if plasma doping, although doping is not very sensitive to stoichiometry in either case. ${ }^{5,13}$

The solid line in Fig. 2 is a fit based on the previously mentioned amphoteric native defect model. ${ }^{7}$ This model predicts that $N_{A} / N_{C}$ is proportional to $\exp \left[2\left(E_{F}-E_{\mathrm{SI}}\right) / k T\right]$, where $N_{A}$ is the concentration of impurity atoms on substitutional sites, $N_{C}$ the concentration of impurities on compensating sites, $E_{F}$ the Fermi level, and $E_{\mathrm{SI}}$ a reference energy level that is constant with respect to the vacuum level for all materials as long as the compensation mechanism is the same. For strong compensation $N_{A} / N_{C}$ is close to one, i.e., $E_{F}$ will approach $E_{\mathrm{SI}}$. The standard relationship between the hole concentration and the Fermi energy allows us then to calculate the maximum obtainable hole concentration at the growth temperature $(550 \mathrm{~K})$ from the relative position of $E_{\mathrm{SI}}$ with respect to the valence band edge. We used $E_{\mathrm{SI}}$ as a parameter to fit the experimental values. Although the carrier concentrations were determined at 300 instead of $550 \mathrm{~K}$, this is justified, since illumination of the samples had no effect on the measured doping levels, showing that nearly all acceptors are activated at room temperature. The best fit was achieved for $E_{\mathrm{SI}}=0.58 \mathrm{eV}$ above the $\mathrm{ZnSe}$ valence band edge. This value is also plotted in Fig. 1, showing that $E_{F}$ at $550 \mathrm{~K}$ lies high in the forbidden gap for Se-rich alloys, while it lies deep in the valence band for Te-rich alloys. As soon as the position of $E_{\mathrm{SI}}$ is deep enough in the band that the resulting carrier concentration approaches the solubility limit of the impurity, $E_{F}$ will be higher than $E_{\mathrm{SI}}$, with the consequence that $N_{A} / N_{C}$ will be large. This means that compensation is negligible and the carrier concentration should be determined only by the solubility. In fact we observe a saturation of the hole concentration at a Te content of $40 \%$, where $E_{F}$ lies 100 $\mathrm{meV}$ below the valence band edge, corresponding to a hole concentration of $10^{20} \mathrm{~cm}^{-3}$. Consequently we interpret this value as the solubility limit of nitrogen in $\mathrm{ZnSe}_{(1-x)} \mathrm{Te}_{(x)}$. A number of further conclusions can be drawn from Fig. 2 .

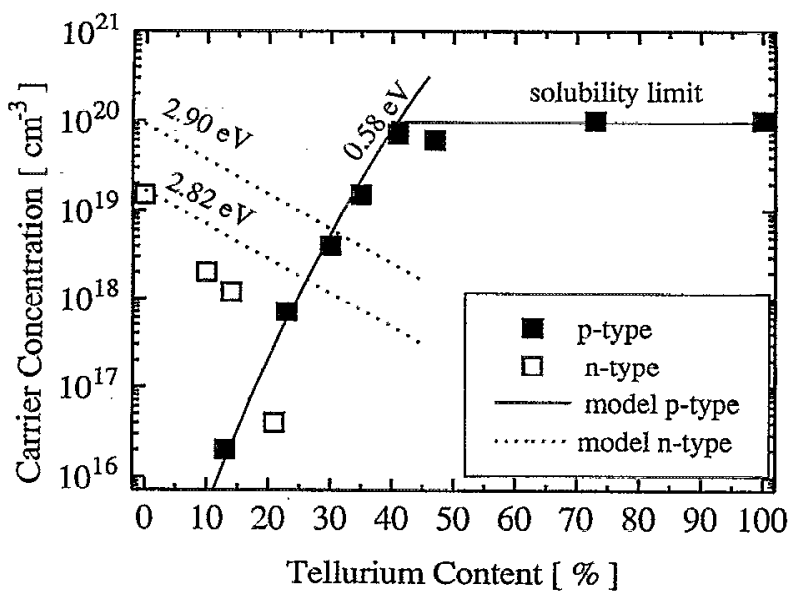

FIC. 2. Obtained $p-$ and $n$-doping levels as a function of the average Te content of $\mathrm{ZnSe} / \mathrm{ZnTe}$ short period SLs (symbols). The lines represent the results of calculations for $p$-type (solid) and $n$-type material (dotted), which depend on the indicated reference energy level $E_{\mathrm{SI}}$. 
(i) In the case of nitrogen doping, even low Te contents lead to a significant improvement of the doping behavior. The good correspondence between the data and the fit shows that the position of the valence band edge with respect to a reference energy level is in fact a key parameter for $p$ doping. If a similar description is valid for $\mathrm{ZnSe}$ doped with if plasma, the model would predict a steep rise in hole concentrations for even lower Te contents.

(ii) Te alloying is especially beneficial for contacting: Even at the lowest measured hole concentration of $2 \times 10^{16}$ (which is near to the detection limit of our Hall apparatus) we obtained good ohmic contacts by evaporating $100 \mathrm{~nm} \mathrm{Au}$ and soldering with In. This is a significant improvement compared to $\mathrm{ZnSe}$, where ohmic contacts cannot even be obtained for the highest possible doping levels. The implication is that the Te content of $\mathrm{ZnSe} T e$ contact layers to $\mathrm{ZnSe}$ lasers could be fabricated with a much lower Te content than previously used. ${ }^{6}$

(iii) For chlorine doping, the addition of Te has a strong negative effect on the obtained carrier concentrations, and above a Te concentration of $20 \%$ we obtain no measurable electron concentration at all. This is in contrast to the predictions of Chadi (Chadi), who assumes a downward bowing of the conduction band of $\mathrm{ZnSeTe}$ mixed crystals, which would result in a higher carrier concentration in the mixed crystal than for $\mathrm{ZnSe}$. The fact that this is not observed is a hint that our calculation of the valence and conduction band variation is more realistic.

The dotted lines in Fig. 2 represent attempts to fit the $n$-type data with the model used for $p$-type material for two different values of $E_{\mathrm{SI}}$. As seen from Fig. 1, the energy shift of the conduction band edge in the composition range below $20 \%$ is much smaller than the corresponding shift of the valence band edge, with the consequence that the slope of the calculated carrier concentration is much smaller for electrons than for holes, and cannot be fitted to the data. Therefore another mechanism, e.g., the formation of deep complexes involving $\mathrm{Cl}$ and $\mathrm{Te}$, must be responsible for the abrupt drop in the carrier concentration. Electron paramagnetic resonance studies are currently on the way to clarify this point.

(iv) There exists no composition range where $p$ - and $n$-doping levels are both high enough for reasonable device applications. The difference in composition between $n$-type and $p$-type material with $10^{18}$ carriers per cubic centimeter is about $10 \%$, corresponding to a lattice mismatch of about $0.8 \%$ between $n$-type and $p$-type material. A way out of this dilemma is the use of the quaternary system
$\mathrm{ZII}_{(1-y)} \mathrm{Mg}_{(y)} \mathrm{Se}_{(1-x)} \mathrm{Te}_{(x)}$ as proposed by Chadi. ${ }^{9}$ In this system, compounds with different Te content can be realized at the same lattice constant. In addition, $\mathrm{Mg}$ has the beneficial effect to compensate for the decrease of the energy gap introduced by the $\mathrm{Te}$, so that the resulting energy gap of the quaternary is in the blue region of the spectrum. Preliminary attempts to realize $p$-type $\mathrm{Zn}_{0.85} \mathrm{Mg}_{0.15} \mathrm{Se}_{0.75} \mathrm{Te}_{0.25}$ showed that the hole concentrations are not affected by a $\mathrm{Mg}$ concentration of $15 \%$, and the luminescence shifts from yellow to blue-green. Since Chadi predicts good $n$ dopability of this system for low Te contents, ${ }^{9}$ we feel that our doping results are encouraging and make detailed studies of the quaternary system attractive.

In conclusion we showed that the addition of $\mathrm{Te}$ to $\mathrm{ZnSe}$ has a strong positive effect on the hole concentration, even at low Te content. Probably the most important effect is that ohmic contacts can easily be formed on $p-\mathrm{ZnSe}_{(1-x)} \mathrm{Te}_{(x)}$. This behavior can be quantitatively understood in terms of the amphoteric native defect model. The opposite holds for $n$ doping: At compositions with good $p$ dopability, the obtained $n$ doping levels are below $10^{16}$. As a way to get structures with both good $p$ - and $n$-doping behavior we propose the use of the quaternary system $\mathrm{Zn}_{(1-y)} \mathrm{Mg}_{(y)} \mathrm{Se}_{(1-x} \mathrm{Te}_{(x)}$.

${ }^{1}$ R. M. Park, M. B. Troffer, C. M. Rouleau, J. M. DePuydt, and M. A. Haase, Appl. Phys. Lett. 57, 2127 (1990).

${ }^{2}$ K. Ohkawa, T. Karasawa, and T. Mitsuyu, J. Cryst. Growth 111, 797 (1991).

${ }^{3}$ M. Haase, J. Qui, J. DePuydt, and H. Cheng, Appl. Phys. Lett. 59, 1272 (1991).

${ }^{4}$ H. Jeon, J. Ding, W. Peatterson, A. V. Nurmikko, W. Xie, D. C. Grillo, M. Kobayashi, and R. Gunshor, Appl. Phys. Lett. 59, 3619 (1991).

${ }^{5}$ J. Han, T. S. Stavrinides, M. Kobayashi, R. L. Gunshor, M. M. Hagerott, and A. V. Nurmikko, Appl. Phys. Lett. 62, 840 (1993).

${ }^{6}$ Y. Fan, J. Han, L. He, J. Saraie, R. L. Gunshor, G. C. Hua, and N. Otsuka, Appl. Phys. Lett. 61, 3160 (1992).

${ }^{7}$ W. Walukiewicz, Materials Science Forum (Trans Tech, Zurich, 1994) Vols. 143-147, p. 519.

${ }^{8}$ C. G. Van der Walle, D. B. Laks, G. F. Neumark, and S. T. Pantelides, Phys. Rev. B 47, 9425 (1993).

${ }^{9}$ D. J. Chadi, Phys. Rev. Lett. 72, 534 (1994).

${ }^{10}$ M. J. S. P. Brasil, M. C. Tamargo, R. E. Nahory, H. L. Gilchrist, and R. J. Martin, Appl. Phys. Lett. 59, 1206 (1991).

${ }^{11}$ Y. Rajakarunanayake, R. H. Miles, G. Y. Yu, and T. C. McGill, Phys. Rev. B 37, 10212 (1988).

${ }^{12}$ F. S. Turco-Sandroff, R. E. Nahory, M. J. S. P. Brasil, R. J. Martin, R. Besermann, L. A. Farrow, J. M. Worlock, and A. L. Weaver, J. Cryst. Growth 111, 762 (1991).

${ }^{13} \mathrm{~S}$. Ferreira, H. Sitter, G. Brunthaler, and W. Faschinger (unpublished).

${ }^{14}$ E. Abramof, K. Hingerl, A. Pesek, and H. Sitter, Semicond. Sci. Technol. 6, A80 (1991).

15 J. Qiu, J. M. DePuydt, H. Cheng, and M. A. Haase, Appl. Phys. Lett. 59, 2992 (1991). 